\title{
An inaccuracy in semi-analytical analysis for Timoshenko beam
}

\author{
Niels Olhoff \\ University of Aalborg, Department of Mechanical Engineering \\ Pontoppidanstraede 101, DK-9220 Aalborg East, Denmark \\ Phone: +4598158522 ext.3513. Fax: +4598151411 \\ and \\ Lucyna Bogdan \\ Systems Research Institute, Polish Academy of Sciences \\ Newelska 6, 01-447 Warsaw, Poland. Phone: 3641 03. Fax: 372772 \\ E-mail: bogdaneibspan.waw.pl
}

\begin{abstract}
In the present paper an exact analysis of the error of sensitivity for a model of Timoshenko beam is considered. The analysis gives a deep insight into the nature of the inaccuracy problem and enables us to devise methods by which the severe error of the sensitivity can be substantially reduced or removed for the model problem.
\end{abstract}

\section{Keywords}

Sensitivity analysis, inaccuracy problem, semi-analytical analysis

\section{INTRODUCTION}

In the present paper the inaccuracy problem through an exact analysis of a model problem is studied. The consideration of the problem is based on the exact analytical solution of the global set of finite element equations for the semi-analytical design sensitivity analysis problem. It is shown that exact sensitivities are obtained for the model problem if the pseudo-loads are computed via exact, analytical differentiation of the stiffness elements with respect to the design variable. If the pseudoloads are determinated via numerical differentiation, the relative error of sensitivity increases with the fourth power of the number of finite elements. 


\section{MODEL PROBLEM}

\section{Finite element setting}

The model problem pertains to Timoshenko beam of constant bending stiffness $E I$ and variable length $L$ that is loaded by a given, concentrated bending moment $M$ at the free end. We have only one design variable $a$ in the model since only the total beam length may vary. In this paper the design variable $a$ will be considered, namely

$a=\ell$, where $\ell=L / n$.

Here $\ell$ is the element length resulting from a uniform subdivision of the beam into $\mathrm{n}$ finite elements. It is the aim of our study to establish expressions for the exact sensitivity $\frac{\delta u_{n}}{\delta L}$ and the approximate sensitivity $\frac{\Delta u_{n}}{\Delta L}$ through finite element analysis using $a=\ell$. We note that $\frac{\delta u}{\delta L}=C_{a} \frac{\delta u}{\delta a} \quad$ where $C_{a}=\frac{1}{n}$.

As Olhoff and Rasmussen (1991a, 1991b) and Cook, Malkus and Plesha (1989) we diskretize the beam into a total number of $\mathrm{n}$ finite elements of equal length, see Figure 1 , $\ell=L / n$. With only one design variable $\ell$, the global equilibrium equations may be written as

$[S(\ell)][D]=[F]$, where $S$ is the stiffness matrix.

Here the nodal displacements $\{\boldsymbol{D}\}$ and external loads $\{\boldsymbol{F}\}$ are defined as

$$
\begin{aligned}
& {[D]=\left[u_{1}, Q_{1}, \ldots, u_{n}, Q_{n}\right]^{T}} \\
& {[F]=\left[p_{1}, M_{1}, \ldots, p_{n}, M_{n}\right]^{T}}
\end{aligned}
$$

where the components $u_{i}, Q_{i}$ in (4) and $p_{i}, M_{i}$ in (5) refer to the right-hand nodal point of the $\mathrm{i}$-th element in the Figure 1.

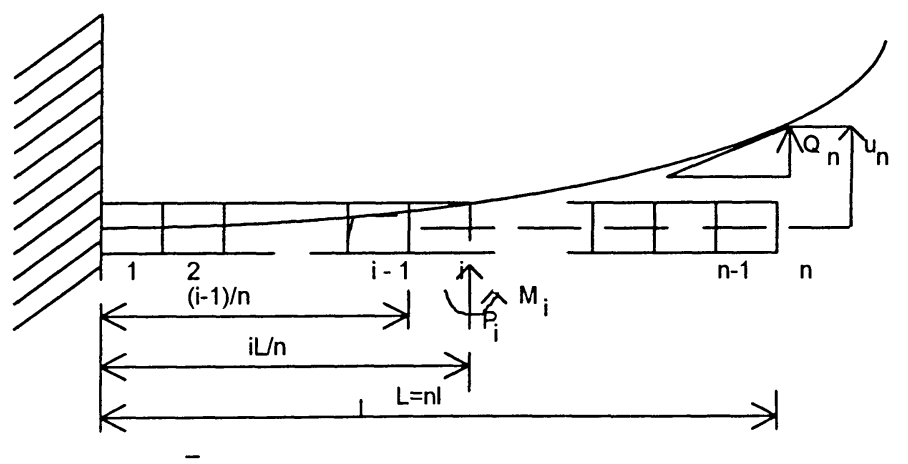

Figure 1 Global finite element model. 
Figure 2 shows the i-th beam element used for the finite element structure in Figure 1, with definitions and sign conventions for the element nodal forces $p_{l i}$ and $p_{2 l}$, moments $m_{l i}$ and $m_{2 i}$, translations $u_{1 i}$ and $u_{2 l}$ and rotations $Q_{1 i}$ and $Q_{2 l}$.

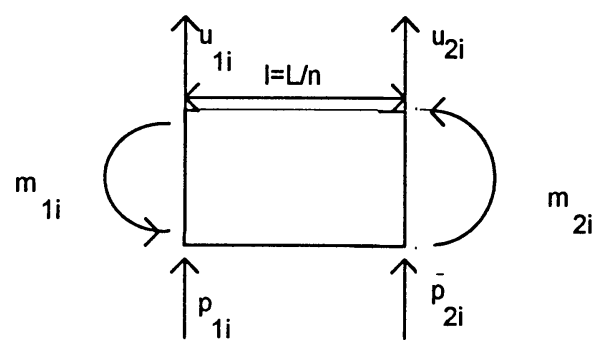

Figure 2 Timoshenko beam element.

The element has the length $\ell$ and bending stiffness $E I$. The element stiffness relations are:

$$
\begin{aligned}
& \left\{\begin{array}{l}
p_{1 i} \\
m_{1 i} \\
p_{2 i} \\
m_{2 i}
\end{array}\right\}=\left[\begin{array}{cccc}
s_{11} & s_{12} & -s_{11} & s_{12} \\
s_{12} & s_{22} & -s_{12} & s_{24} \\
-s_{11}-s_{12} & s_{11}-s_{12} \\
s_{12} & s_{24} & -s_{12} & s_{22}
\end{array}\right]\left\{\begin{array}{l}
u_{1 i} \\
Q_{1 i} \\
u_{2 i} \\
Q_{2 i}
\end{array}\right\} \\
& =\left[\begin{array}{llll}
p_{11}+r_{11} & p_{12}+r_{12}-p_{11}-r_{11} & p_{12}+r_{12} \\
p_{12}+r_{12} & p_{22}+r_{22} & -p_{12}-r_{12} & p_{22}+r_{24} \\
-p_{11}-r_{11} & -p_{12}-r_{12} & p_{11}+r_{11} & -p_{12}-r_{12} \\
p_{12}+r_{12} & p_{22}+r_{24}-p_{12}-r_{12} & p_{22}+r_{22}
\end{array}\right] *\left\{\begin{array}{l}
u_{1 i} \\
Q_{1 i} \\
u_{2 i} \\
Q_{2 i}
\end{array}\right\}
\end{aligned}
$$

where the values of $p_{11}, p_{12}, p_{22}$ and $r_{11}, r_{12}, r_{22}$ and $r_{24}$ are given by

$$
\begin{aligned}
& p_{11}=K A G \frac{1}{\ell}(1-\gamma)^{2}, \quad p_{12}=\frac{1}{2} \operatorname{KAG}(1-\gamma)^{2}, \quad p_{22}=K A G \frac{\ell}{4}(1-\gamma)^{2}, \quad r_{I I}=E I \frac{12 \gamma^{2}}{\ell^{3}}, \\
& r_{I 2}=6 E I \frac{\gamma^{2}}{\ell^{2}}, \quad r_{22}=E I \frac{1+3 \gamma^{2}}{\ell}, \quad r_{24}=E I \frac{3 \gamma^{2}-1}{\ell} .
\end{aligned}
$$

The coefficient $\gamma$ can be computed as

$$
\gamma=\frac{1}{1+12 \frac{E I}{K A G \ell^{2}}}
$$

$\mathrm{KAG}$ is the shear stiffness. 
From the expression (6) and according to the relations

$u_{2 i}=u_{1 i+1}, \quad u_{1 i}=u_{2 i-1}, \quad Q_{1 i}=Q_{2 i-1}, \quad Q_{2 i}=Q_{1 i+1}$ we obtain:

$\left.\begin{array}{l}-s_{11} u_{i-1}-s_{12} Q_{i-1}+2 s_{11} u_{i}-s_{11} u_{i+1}+s_{12} Q_{i+1}=P_{i} \\ s_{12} u_{i-1}+s_{24} Q_{i-1}+2 s_{22} Q_{i}-s_{12} u_{i+1}+s_{24} Q_{i+1}=M_{i}\end{array}\right\}$ for $\mathrm{i}=1, \ldots, \mathrm{n}-1$

$\left.\begin{array}{l}-s_{11} u_{n-1}+s_{12} Q_{n-1}+s_{11} u_{n}-s_{12} Q_{n}=P_{n} \\ s_{12} u_{n-1}+s_{24} Q_{n-1}-s_{12} u_{n}+s_{22} Q_{n}=M_{n}\end{array}\right\} \quad$ for $\mathrm{n}$.

By means of (8) we can easily identify the form of the global stiffness matrix $[S]$ in (3) in terms of the element stiffness components $s_{11}, s_{12}, s_{22}$ and $s_{24}$ defined in (6). Now, assuming the components $u_{i}, Q_{i}, i=1, \ldots, n$ of the model displacement vector $[D]$ in (3) and (9) to be known subject to a given model load vector $[F]$ we may write $(9)$ in the form

$P_{i}=s_{11} f_{i 1}+s_{12} f_{i 2}$
$M_{i}=s_{12} g_{i 1}+s_{22} g_{i 2}+s_{24} g_{i 4}$

where, for example, the coefficient $f_{i l}$ is given by

$f_{i 1}=-u_{i-1}+2 u_{i}-u_{i+1}$

and so on.

\section{Analytical sensitivity analysis problem statement}

Let us denote by $\delta\{D\} / \delta l$ the vector of model displacement sensitivities with respect to the design variable $\ell$, where $\ell=\frac{L}{n}$. This vector of sensitivities constitutes the solution to the equation:

$[S(\ell)] \frac{\partial[D]}{\partial \ell}=[F]_{\partial \ell}$

which we obtain by analytical differentiation of the equilibrium equations (3) with respect to the design variable $\ell$. Differentiating the expression (3) we obtain

$\frac{\partial S}{\partial \ell} D+S \frac{\partial D}{\partial \ell}=\frac{\partial F}{\partial \ell}$.

Because the external load is independent on the design, so $\frac{\partial F}{\partial \ell}=0$ and

$[F]_{\partial \ell}=[S(\ell)] \frac{\partial[D]}{\partial \ell}=-\frac{\partial S(\ell)}{\partial \ell}[D] .\{F\}_{\partial \ell}$ in (12) is termed pseudo load vector and it has a form:

$[F]_{\partial \ell}=\left\{\left[P_{1 \partial \ell}, M_{1 \partial \ell}, \ldots, P_{n \partial \ell}, M_{n \partial \ell}\right]\right\}^{T}$. 
From the analogy between (12) and (3) and the equivalent form (10) and (3) it follows, that the components of the pseudo load vector $[F]_{\partial \ell}$ in (12) and (14) can be computed as

$$
\begin{aligned}
& P_{i \partial \ell}=-\frac{\partial s_{11}}{\partial \ell} f_{i 1}-\frac{\partial s_{12}}{\partial \ell} f_{i 2} \\
& M_{i \partial \ell}=-\frac{\partial s_{12}}{\partial \ell} g_{i 1}-\frac{\partial s_{22}}{\partial \ell} g_{i 2}-\frac{\partial s_{24}}{\partial \ell} g_{i 4} \quad i=1, \ldots, n
\end{aligned}
$$

\section{Semi-analytical problem statement}

The only difference between the analytical and the semi-analytical sensitivity analysis problems is that while the pseudo loads are determined via analytical differentiation of the stiffness matrix in the former, in the latter they are obtained via numerical differentiation. The pseudo load vector

$$
\{F\}_{\Delta \ell}=\left\{P_{1 \Delta \ell}, M_{1 \Delta \ell}, \ldots, P_{n \Delta \ell}, M_{n \Delta \ell}\right\}^{T}
$$

is based on approximate differentiation of the stiffness matrix. The components of $\{F\}_{\Delta \ell}$ can then be computed as:

$$
\begin{aligned}
P_{i \Delta \ell} & =-\frac{\Delta s_{11}}{\Delta \ell} f_{i 1}-\frac{\Delta s_{12}}{\Delta \ell} f_{i 2} \\
M_{i \Delta \ell} & =-\frac{\Delta s_{12}}{\Delta \ell} g_{i 1}-\frac{\Delta s_{22}}{\Delta \ell} g_{i 2}-\frac{\Delta s_{24}}{\Delta \ell} g_{i 4} \quad i=1, \ldots, n
\end{aligned}
$$

Exact solutions to the finite element equations for displacements and design sensitivities

The well-known Bernoulli-Euler transverse deflection and slope for this problem are given by

$u(x)=\frac{M x^{2}}{2 E I} ; \quad Q(x)=u^{\prime}(x)=\frac{M x}{E I}$

In the finite element setting (18) can be expressed as

$u_{i}(M)=\frac{M L^{2}}{2 E I}\left(\frac{i}{n}\right)^{2}$
$Q_{i}(M)=\frac{M L}{E I} \frac{i}{n}, \quad i=0, \ldots, n$

Now we can determine the expressions $f_{i l}, f_{i 2}, f_{n l}, f_{n 2}, g_{i l}, g_{i 2}, g_{i 4}, g_{n l}, g_{n 2}, g_{n 4}$ in (10). For example

$$
\begin{aligned}
& f_{i 1}=-u_{i-1}+2 u_{i}-u_{i+1}=-\frac{M L^{2}}{E I} \frac{1}{n^{2}} \\
& f_{i 2}=-Q_{i-1}+Q_{i+1}=2 \frac{M L}{E I} \frac{1}{n}
\end{aligned}
$$


So let us solve analytically the beam equation for the deflection $u_{n}$ at the beam end subject to a given concentrated force $P_{i}$ and moment $M_{i}$. Then let us apply the superposition principle to obtain the displacement $u_{n}$ subject to the application of the external loads $P_{i}, M_{i}$ in all nodal points $\mathrm{i}=1, \ldots, \mathrm{n}$. We have

$$
\begin{aligned}
& u_{n}=\sum_{i=1}^{n} u\left(M_{i}\right)+\sum_{i=1}^{n} u\left(P_{i}\right)=\sum_{i=1}^{n}\left[\frac{M_{i} L^{2}}{2 E I}\left(\frac{i}{n}\right)\left(2-\frac{i}{n}\right)\right]+\sum_{i=1}^{n} P_{i}\left[K A G L+\frac{L^{3}}{6 E I}\left(\frac{i}{n}\right)^{2}\left(3-\frac{i}{n}\right)\right]= \\
& =L\left\{\sum_{i=1}^{n} M_{i} \frac{L}{2 E I}\left(\frac{i}{n}\right)\left(2-\frac{i}{n}\right)+P_{i}\left[K A G+\frac{L^{2}}{6 E I}\left(\frac{i}{n}\right)^{2}\left(3-\frac{i}{n}\right)\right]\right\}
\end{aligned}
$$

From the analogy between (3) and (12) it follows, that

$$
\frac{\partial u_{n}}{\partial \ell}=L \sum_{i=1}^{n}\left[p_{i \partial \ell}\left[K A G+\frac{L^{2}}{6 E I}\left(\frac{i}{n}\right)^{2}\left(3-\frac{i}{n}\right)\right]+M_{i \partial \ell} \frac{L}{2 E I}\left(\frac{i}{n}\right)\left(2-\frac{i}{n}\right)\right]
$$

and similarly the component $\frac{\Delta u_{n}}{\Delta \ell}$ is determined by:

$$
\frac{\partial u_{n}}{\Delta \ell}=L \sum_{i=1}^{n}\left[p_{i \Delta \ell}\left[K A G+\frac{L^{2}}{6 E I}\left(\frac{i}{n}\right)^{2}\left(3-\frac{i}{n}\right)\right]+M_{i \Delta \ell} \frac{L}{2 E I}\left(\frac{i}{n}\right)\left(2-\frac{i}{n}\right)\right]
$$

\section{PSEUDO LOADS AND DESIGN SENSITIVITY OBTAINED}

\section{BY ANALYTICAL DIFFERENTIATION OF THE STIFFNESS}

\section{MATRIX}

We have calculated the values of expressions $s_{11}, s_{l 2}, s_{22}, s_{24}$ and $\frac{\partial s_{11}}{\partial \ell}, \frac{\partial s_{12}}{\partial \ell}, \frac{\partial s_{22}}{\partial \ell}, \frac{\partial s_{24}}{\partial \ell}$.

Using these values we obtain:

$$
\begin{aligned}
& P_{i \partial \ell}=-\frac{12 A G K M n^{2}}{A G K L^{2}+12 E I n^{2}} \\
& M_{i \partial \ell}=-\frac{12 A G K L n i}{A G K L^{2}+12 E I n^{2}} \\
& P_{n \partial \ell}=\frac{6 A G K M n^{2}(2 n-1)}{A G K L^{2}+12 E I n^{2}} \\
& M_{n \partial \ell}=-\frac{2 M n\left(A G K L^{2}(3 n-2)-6 E I n^{2}\right)}{L\left(A G K L^{2}+12 E I n^{2}\right)}
\end{aligned}
$$


Now we can obtain the value of expression (22)

$\frac{\partial u_{n}}{\partial \ell}=\frac{L M\left(A G K L^{2}+6 E I n(n+1)\right)}{E I\left(A G K L^{2}+12 E I^{2}\right)}$

We can notice, that if $\mathrm{K} \rightarrow \infty$ we have

$\lim _{K \rightarrow \infty} \frac{\partial u_{n}}{\partial \ell}=\lim _{K \rightarrow \infty} \frac{L M}{E I} \frac{A G L^{2}+\frac{6 E I n(n+1)}{K}}{A G L^{2}+\frac{12 E I^{2}}{K}}=\frac{L M}{E I}$

The result (26) is the same as the exact result in the case of Bernoulli-Euler beam.

\section{EXACT ERROR ANALYSIS OF THE SEMI -ANALYTICAL SENSITIVITY PROBLEM}

We now focus on the semi-analytical sensitivity analysis problem stated in section 2, adopting first -order forward difference approximations to the derivatives of the stiffness components that constitute the basis for the computation of the approximate pseudo-load vector $[F]_{\Delta \ell}$.Thus we obtain the following expressions for the forward finite difference approximations to the derivatives of stiffness. We use the relation

$$
-\frac{\Delta s_{i j}}{\Delta \ell}=\frac{s_{i j}(\ell)-s_{i j}(\ell+\Delta \ell)}{\Delta \ell} \text { for } \mathrm{i}=1,2 \text { and } \mathrm{j}=1,2,4
$$

So we obtain the expressions $-\frac{\Delta s_{11}}{\Delta \ell},-\frac{\Delta s_{12}}{\Delta \ell},-\frac{\Delta s_{22}}{\Delta \ell},-\frac{\Delta s_{24}}{\Delta \ell}$. We may now express in a similar fashion the approximate pseudo load components $P_{i \Delta \ell}, P_{n \Delta \ell}, M_{i \Delta \ell}$ and $M_{n \Delta \ell}$.

$$
\begin{aligned}
P_{i \Delta \ell} & =-\frac{12 K A G L M n^{2}}{\left(K A G((\Delta \ell) n+L)^{2}+12 E I^{2}\right)((\Delta \ell) n+L)} \\
P_{n \Delta \ell} & =\frac{6 K A G L M n^{2}(2 n-1)}{\left(K A G((\Delta \ell)+L)^{2}+12 E I^{2}\right)((\Delta \ell) n+L)} \\
M_{i \Delta \ell} & =-\frac{12 K A G L M n i}{K A G((\Delta \ell) n+L)^{2}+12 E I n^{2}} \\
M_{n \Delta \ell} & =\frac{M n\left(K A G((\Delta \ell) n-2 L(3 n-2))((\Delta \ell) n+L)+12 E I n^{2}\right)}{\left(K A G((\Delta \ell) n+L)^{2}+12 E I n^{2}\right)((\Delta \ell) n+L)}
\end{aligned}
$$

Now we may calculate the semi-analytical displacement sensitivity $\frac{\Delta u_{n}}{\Delta L}$ that is the subject of our model problem: 


$$
\frac{\Delta u_{n}}{\Delta L}=\frac{L^{2} M\left[K A G\left((\Delta \ell)^{2} n^{2}-(\Delta \ell) L n\left(5 n^{2}-4\right)+2 L^{2}\right)+12 E I n(n+1)\right]}{2 E I\left[K A G((\Delta \ell) n+L)^{2}+12 E I n^{2}\right]((\Delta \ell) n+L)}
$$

Let us now establish the expression for the relative error

$$
\varepsilon=\frac{\frac{\Delta u}{\Delta L}-\frac{\partial u}{\partial L}}{\frac{\partial u}{\partial L}}
$$

We have obtained the result

$$
\varepsilon=-\frac{\eta\left(K^{2} A^{2} G^{2} L^{4}\left(5 n^{2}+2 \eta^{2}+5 \eta+2\right)+12 K A G E I L^{2} n\left(5 n^{3}+n\left(\eta^{2}+2 \eta+1\right)+\eta^{2}+3 \eta+3\right)\right.}{2\left(K A G L^{2}+6 E \operatorname{In}(n+1)\right)\left(K A G L^{2}(\eta+1)^{2}+12 E I n^{2}(\eta+1)\right)}
$$

where $\eta=\frac{\Delta \ell}{\ell}$

As we may see $\varepsilon$ is the function of $n^{4}$ and $\eta^{2}$, not $n^{2}$ and $\eta$, as it has been for Bernoulli-Euler case. But we may notice, that

$$
\lim _{n \rightarrow \infty} \varepsilon=\frac{-\eta\left(5 K A G L^{2}+12 E I\right)}{12 E I(\eta+1)}
$$

This means that the error $\varepsilon$ of the semi-analytical displacement sensitivity does not increase to infinity if $\mathrm{n}$ increases to infinity. We have tried to calculate also the boundary of $\frac{\Delta u}{\Delta L}$ for $\mathrm{K} \rightarrow \infty$ and $\Delta \ell \rightarrow 0$. This result is $\frac{L M}{E I}$. As we can notice it is the same as for Bernoulli-Euler beam.

\section{REFERENCES}

Cook, R.D., Malkus, D.S. and Plesha, M.E. (1989) Concepts and applications of finite element analysis. Wiley \& Sons, New York.

Olhoff, N. and Rasmussen, J. (1991a) Method of error elimination for a class of semianalytical sensitive analysis problems, in Engineering optimization in design processes, (ed. Eschenauer, H.A., Mattheck, C., Olhoff, N.) pp.193-200, Springer, Berlin, Heidelberg, New York.

Olhoff, N. and Rasmussen, J. (1991b) Study of inaccuracy in semi-analytical sensitivity analysis a model problem. Structural Optimization, 3, 203-213. 